Ergod. Th. \& Dynam. Sys. (1988), 8, 53-72

Printed in Great Britain

\title{
An invariant for rigid rank-1 transformations
}

\author{
NATHANIEL FRIEDMAN, PATRICK GABRIEL \\ AND JONATHAN KING
}

Department of Mathematics and Statistics, SUNY at Albany, Albany, NY 12222, USA; Laboratoire de Probabilités, Université de Dijon, Dijon, France; Department of Mathematics, University of Maryland, College Park, MD 20742, USA

(Received 6 June 1986 and revised 6 February 1987)

Abstract. Associated to a rigid rank-1 transformation $T$ is a semigroup $\mathscr{L}(T)$ of natural numbers, closed under factors. If $\mathscr{L}(S) \neq \mathscr{L}(T)$ then $S$ and $T$ cannot be copied isomorphically onto the same space so that they commute. If $\mathscr{L}(S) \not \supset \mathscr{L}(T)$ then $S$ cannot be a factor of $T$. For each semigroup $L$ we construct a weak mixing $S$ such that $\mathscr{L}(S)=L$. The $S$ where $\mathscr{L}(S)=\{1\}$, despite having uncountable commutant, has no roots.

Preceding and preparing for this example are two others: An uncountable abelian group $G$ of weak mixing transformations for which any two (non-identity) members have identical self-joinings of all orders and powers. The second example, to contrast with the rank-1 property that the weak essential commutant must be the trivial group, is of a rank-2 transformation with uncountable weak essential commutant.

The purpose of this note is to explore some of the consequences and limitations of the weak-closure theorem of [3].

The commutant group, $C(T)$, of a transformation $T$ has as a subgroup of its center, the closure in the weak topology of its powers $\left\{T^{k}\right\}_{k=-\infty}^{\infty}$. Denote this weak closure subgroup by $\mathrm{WCl}(T)$. The weak-closure theorem says that for rank-1 transformations $\mathrm{WCl}(T)=C(T)$. A dichotomy, then, exists in the class Rank-1: $\mathrm{C}(T)$ is uncountable or is trivial (just the powers of $T$ ) depending on whether $T$ is rigid or not. Ornstein's rank-1 mixing transformation and Chacon's transformation are evidently on the non-rigid side of the dichotomy. On the rigid side, the commutant group is not well understood either in terms of the types of transformations that can appear in it (prime transformations can, [1]; positive entropy can; finite rank mixing cannot, [4]) nor its structure as a group. One can work from two sides: Building examples, or exhibiting properties which inherit under weak limits.

All examples constructed herein are weak mixing. Definitions are in $\S 0$ along with a disjointedness result for a direct product which is rank-1.

$\S 1$ constructs an uncountable group of commuting rank-1 transformations. Each such is of necessity a weak limit of powers of any other and so they all have the same self-joining measures of all orders and powers. 
Define two quotient groups, the essential commutant and the weak essential commutant by

$$
\operatorname{EC}(T)=C(T) /\left\{T^{k}\right\}_{-\infty}^{\infty} \quad \operatorname{WEC}(T)=C(T) / \mathrm{WCl}(T) .
$$

In $\S 2$ we build a rank-2 $T$ such that $\operatorname{WEC}(T)$ is uncountable.

The title of this paper comes from $\S 3$. Therein is a rank-1 $S$ for which

$$
\begin{array}{ll}
\operatorname{rk}\left(S^{\prime}\right)=1 & l \text { odd; } \\
\operatorname{rk}\left(S^{l}\right)=2 &
\end{array}
$$

The oscillation of the function $l \mapsto \mathrm{rk}\left(S^{\prime}\right)$ gives rise to the invariant $\mathscr{L}(\cdot)$ of the abstract.

$\S 0$. Consider the class $\mathscr{T}$ of transformations on a fixed Lebesgue probability space $(X, A, \mu)$. A usual natural topology on $\mathscr{T}$ is that of weak convergence on sets. For $\left\{T_{n}\right\} \subset \mathscr{T} \ni S$ say that $T_{n} \rightarrow S$ or $S=\lim _{n} T_{n}$ if, for each set $B \in \mathscr{A}$

$$
\mu\left(T_{n}^{-1} B \Delta S^{-1} B\right) \underset{n}{\rightarrow} 0
$$

This topology can be realized by a (non-canonical) metric $\mathbf{m}(\cdot, \cdot)$ by choosing a countable generating sub-algebra $\left\{A_{1}, A_{2}, \ldots\right\}$ of $\mathscr{A}$ and defining

$$
\mathbf{m}(T, S)=\sum_{k=1}^{\infty} \mu\left(T^{-1} A_{k} \Delta S^{-1} A_{k}\right) / 2^{k}
$$

Under $\mathbf{m}, \mathscr{T}$ is a complete separable metric space. If $T_{n} \rightarrow S$ and each $T_{n}$ is invertible, the limit $S$ will in general not be invertible. However if all the $\left\{T_{n}\right\}$ commute with each other, their limit must be invertible.

Alternatively, should one wish to restrict $\mathscr{T}$ to only invertible transformations, one can still realize the weak topology by a metric under which $\mathscr{T}$ is complete by replacing the lefthand side of $(0.1)$ by

$$
\left[\mu\left(T_{n}^{-1} B \Delta S^{-1} B\right)+\mu\left(T_{n}(B) \Delta S(B)\right)\right] / 2
$$

along with the analogous modification in $\left(0.1^{\prime}\right)$. For us, the distinction is moot as we will consider weak limits where all the $\left\{T_{n}\right\}$ are various powers of the same invertible transformation and hence mutually commute. Note that under the weak topology the invertible transformations form a topological group.

A transformation $T$ is called rigid if there exists $\left\{k_{n}\right\}$, a never-zero sequence of rigidity times, such that $T^{k_{n}} \rightarrow \mathrm{Id}$, where Id denotes the identity transformation.

We will denote the (non-uniform) rank of $T$ by $\mathrm{rk}(T)$. The ' $\varepsilon$-refining' definition of rank is the infimum of integers $r$ such that, given any $\varepsilon$ and any partition $Q$ : There exists $r$ disjoint Rohlin stacks in the space such that $Q$ is $\varepsilon$-refined by the partition whose atoms are the column levels of the stacks, and the complement. We will also make use, in $\S 2$, of a well known equivalent definition of rank that uses names. For a precise definition see [4] or [3], but roughly it says that any name can be mostly covered by copies of $r$ words. By this 'name' characterization of rank one shows rk $(F) \leq \mathrm{rk}(T)$ for any factor $F$ of $T$.

For idiosyncrasies we use $a \triangleq b$ (or $b \unrhd a$ ) to mean that the expression $b$ defines the (new) symbol $a$. Also, when wishing to indicate the sigma-algebra or the measure 
on which acts a transformation $T$, we may write

$$
(T: X, \mathscr{A}, \mu)
$$

in lieu of the longer $T:(X, \mathscr{A}, \mu) \rightarrow(X, \mathscr{A}, \mu)$. If the algebra is understood we write $(T: X, \mu)$.

Definition. Say that a transformation $T$ is commutant dense if $\mathrm{WCl}(T)=C(T)$. Let $\mathrm{CD}$ denote the class of such transformations. A commutant dense transformation has abelian commutant. The weak-closure theorem asserts

$$
\text { Rank-1 } \subset \mathrm{CD}
$$

but, outside of this, the relation between these two classes is unknown, for rigid transformations. For non-rigids, CD is strictly larger than Rank-1. One can use an automorphism extension (defined in \$3) of Ornstein's rank-1 mixing to make a rank-3 mixing transformation with trivial commutant. This is done in [4].

Joinings. Given a countable collection of transformations $\left(S_{l}: X_{l}, \mu_{l}\right)$ let

$$
\text { Joi }\left(S_{1}, S_{2}, \ldots, S_{l}, \ldots\right)
$$

denote the collection of joinings, that is, of measures on the product space $X_{1} \times X_{2} \times$ $\cdots$ invariant under $S_{1} \times S_{2} \times \cdots$ and with the correct 1-dimensional marginal measures $\mu_{1}, \mu_{2}, \ldots$.

The $\left\{S_{l}\right\}$ are said to be collectively disjoint (in the sense of Furstenberg) if the only joining of $\left\{S_{1}, S_{2}, \ldots\right\}$ is product measure $\mu_{1} \times \mu_{2} \times \cdots$.

Suppose we have transformations ${ }_{n} T \stackrel{n}{\rightarrow} T$ (in the weak topology) on some space $X$, and ${ }_{n} R \stackrel{n}{\rightarrow} R$ on some other space $Y$. If $\nu(\cdot)$ is a measure on $X \times Y$ which happens to be a joining of ${ }_{n} T$ with ${ }_{n} R$ for each $n$, then the condition of correct marginals implies that $\nu$ is $T \times R$-invariant. This is of course true for joinings of countably many transformations.

If $S_{l}$ and ${ }_{n} S_{l}$ are transformations on $X_{l}$ with ${ }_{n} S_{l} \rightarrow S_{l}$ as $n \rightarrow \infty$, then

$$
\left[\forall n: \nu \in \operatorname{Joi}\left({ }_{n} S_{1},{ }_{n} S_{2}, \ldots\right)\right] \Rightarrow \nu \in \operatorname{Joi}\left(S_{1}, S_{2}, \ldots\right) \text {. }
$$

Thus joinings are inherited under weak limits.

In [3] it was shown by separate arguments that for a rank-1 $T$

(a) $T$ cannot be of the form $S^{n} \times S^{m}$.

(b) If $T=S_{1} \times S_{2}$ then $S_{1}$ and $S_{2}$ are disjoint.

It turns out that both (a) and (b) are consequences of a stronger disjointedness property.

Rudolph and del Junco, [2], use 'power joining' for a joining between (possibly different) powers of a transformation. We adopt this terminology and say that the transformations of a countable list $\left\{S_{l}\right\}$ are collectively power disjoint if: For any sequence of integers $\left\{r_{l}\right\}$ such that each $\left(S_{l}\right)^{r_{l}}$ is ergodic, the transformations $\left\{S_{l}^{r_{l}}\right\}$ are collectively disjoint.

THEOREM 0.3. If a direct product $T=S_{1} \times S_{2} \times \cdots$ is commutant dense (in particular, if it is rank-1), then the $\left\{S_{l}\right\}$ are collectively power disjoint.

Remark. This is non-vacuous; a weak-mixing rank-1 countable direct product appears in [3]. 
Proof. We use the observation of [2] that the identity transformation is disjoint from any ergodic transformation.

Denote the space $S_{l}$ acts on by $\left(X_{l}, \mu_{l}\right)$. Fix exponents $\left\{r_{l}\right\}$, set $R_{l} \triangleq S_{l}^{r_{l}}$, and choose $\nu \in \mathrm{Joi}\left(R_{1}, R_{2}, \ldots\right)$. Let $\nu_{l}$ denote the joining of $\left\{R_{1}, \ldots, R_{l}\right\}$ which is $\nu$ restricted to (projected onto) $X_{1} \times \cdots \times X_{l}$. It suffices to show that for each $l$, $\nu_{l}=\mu_{1} \times \cdots \times \mu_{l}$. By induction then, we need but show that $\nu_{L}=\nu_{L-1} \times \mu_{L}$ for each fixed $L$.

Let $I_{I}$ denote the identity transformation on $X_{I}$. The transformation

$$
I_{1} \times \cdots \times I_{L-1} \times S_{L} \times I_{L+1} \times \cdots
$$

commutes with $T$ and is consequently the weak limit of some sequence of powers $T^{k_{n}}$. Hence as $n \rightarrow \infty: S_{l^{n}}^{k_{n}} \rightarrow S_{L}$ and for those $l \neq L, S_{l}^{k_{n}} \rightarrow I_{l}$. Raising the $l$ th limit to the power $r_{l}$ we conclude that as $n \rightarrow \infty: R_{l}^{k^{n}} \rightarrow I_{l}$, for $1 \leq l<L$, and $R_{L^{n}}^{k^{\prime \prime}} \rightarrow R_{L}$. So by $(0.2)$

$$
\nu_{L} \in \operatorname{Joi}\left(I_{1}, \ldots, I_{L-1}, R_{L}\right)
$$

Disjointness of the identity transformation $I_{1} \times \cdots \times I_{L-1}$ from the ergodic transformation $R_{L}$, implies $\nu_{L}=\nu_{L-1} \times \mu_{L}$ as desired.

Remark 0.4. Given an $(S: X, \mu)$, an integer vector $\left\langle n_{1}, n_{2}, \ldots\right\rangle$ and any $\rho \in$ Joi $\left(S^{n_{1}}, S^{n_{2}}, \ldots\right)$ then

$$
\left(S^{n_{1}} \times S^{n_{2}} \times \cdots: X^{\mathbb{N}}, \rho\right)
$$

is itself a transformation. Agree to call this transformation a 'power-joining of $S$ '.

The argument of the preceding theorem yields an apparently stronger disjointedness, whose proof we leave to the reader. For want of a better name, say that the sequence $\left\{S_{l}\right\}_{1}^{x}$ is very disjoint if $\left\{R_{l}\right\}_{1}^{x}$ is collectively disjoint whenever: each $R_{l}$ is an ergodic power-joining of $S_{l}$.

The assertion: If $\left\{S_{l}\right\}$ are such that $S_{1} \times S_{2} \times \cdots$ is commutant dense, then the $\left\{S_{l}\right\}$ are very disjoint.

1. An uncountable abelian group of weak mixing transformations with identical power joinings

Suppose that $\nu$ is a joining of various powers of a single transformation, what the authors of [2] call a 'power joining'.

$$
\nu \in \operatorname{Joi}\left(T^{r_{1}}, T^{r_{2}}, \ldots, T^{r_{l}}, \ldots\right) \quad r_{l} \in \mathbb{Z} \text {. }
$$

If $S$ is a weak limit of powers $T^{k_{n}}$ then $\left(T^{r_{i}}\right)^{k_{n}} \rightarrow_{n} S^{r_{l}}$ and hence

$$
\nu \in \text { Joi }\left(S^{r_{1}}, S^{r_{2}}, \ldots, S^{r_{1}}, \ldots\right) \text {. }
$$

Thus the power joinings of $T$ are seen to be hereditary under weak limits; they pass to every $S \in \mathrm{WCl}(T)$. In particular, $S$ inherits all the factor algebras of $T$ ( $S^{2}$ inherits those of $T^{2} ; S^{3}$, of $T^{3}$ etc.) as well as the commutant of $T$ i.e. $C(S) \supset C(T)$ and similarly for corresponding powers. [Note in passing that the property 'presence of a rotation factor' inherits under weak limits; for if $\left.T\right|_{\mathscr{F}}$ is (isomorphic to) a rotation on the circle, where $\mathscr{F}$ denotes some factor algebra, then $\left.S\right|_{\mathscr{F}}$ is a weak limit of powers of this rotation and hence is, itself, a rotation. Thus, we may conclude 
that lack of weak mixing inherits under weak limits, since 'lack of ergodicity' certainly does.]

Returning, suppose now that our limit $S$ happens to have $T \in \mathrm{WCl}(S)$. Since the inheritance now goes the other way, $T$ and $S$ have exactly the same power joinings:

$$
\text { Joi }\left(T^{r_{1}}, T^{r_{2}}, \ldots\right)=\text { Joi }\left(S^{r_{1}}, S^{r_{2}}, \ldots\right)
$$

for each integer vector $\left\langle r_{1}, r_{2}, \ldots\right\rangle$.

Summing up, we can fulfill the title of this section by constructing a rigid weak mixing $T$ and an uncountable group $G, G \subset \mathrm{WCl}(T)$, such that each (non-identity) $S \in G$ is rank-1 (hence has $T$ in its weak closure group). That such an $S$ is weak mixing - although a consequence of the above remark in brackets - follows on general principles because, as A. del Junco points out, any ergodic transformation which commutes with a weak mixing, must itself be weak mixing.

We now build $T$ and $G$.

Construction. We build a rank-1 $T$ via cutting and stacking, letting $h_{n}$ and $B_{n}$ denote, respectively, the height and the base set, of the $n$-stack. It being irrelevant how you start, we describe the induction step.

At stage $N$ we define the $(N+1)$-block in terms of the $N$-block by choosing a $b \in \mathbb{N}$ and an $s \in\left[0, f_{N}\right)$; here $f_{N}$ is a specific function of the numbers $h_{1}, \ldots, h_{N}$ and $N$, and will be described further on.

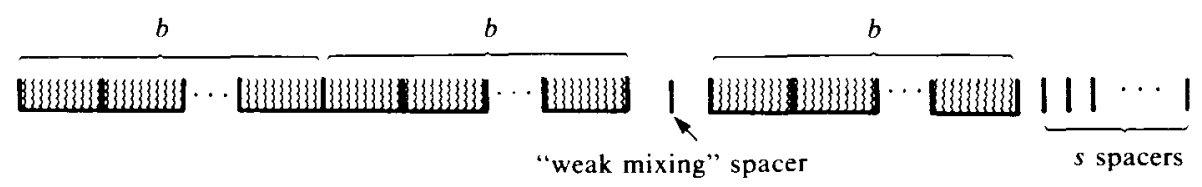

Figure 1.1. The $(N+1)$-block consists of $2 b$ many $N$-blocks, then a spacer, then $b$ more $N$-blocks, and ending with $s$ spacers. Thus $h_{N+1}=3 b h_{N}+(s+1)$.

To get the form of this $T$ we have dusted off the transformation of example $(v)$ in [3] which has all of its powers rank-1. This time, we manipulate parameters $b$ and $s$ so that rigidity is added with speed sufficient for rank-1-ness to pass to some of the weak limits. The choice of how to present the proof is idiosyncratic: The two ideas are that, since we may examine the $N$-stack before choosing the $b$ and $s$ which determine the $(N+1)$-stack

(i) One can make $h_{N+1}$ relatively prime to all natural numbers less than some specified function of $h_{1}, \ldots, h_{N}$.

(ii) An upper bound on $\mu\left(B_{N} \Delta T^{h_{N}}\left(B_{N}\right)\right) / \mu\left(B_{N}\right)$ may be chosen depending on $h_{N}$ and $N$.

First we check the basics. The number $f_{N} / h_{N+1}$ upper bounds the fraction of the $(N+1)$-stack which is spacer. Since, at stage $N$, we can make $b$ sufficiently large that $f_{N} / h_{N+1}$ is a summable function of $N$; so $T$ is indeed defined on a probability space. Moreover, due to the 'weak mixing' spacer in the figure, the usual Chacón argument shows that $T$ has no eigenvalues. Since $T$ is rank-1, hence ergodic, $T$ is weak mixing. 
We may now put arbitrary constraints on how at stage $N$ to choose $b$ and $s$, so long as the restrictions placed on $b$ allow it to be chosen 'sufficiently large'.

How to choose the parameters. Note that for any positive integer $L$ :

$\forall d \in \mathbb{Z}$ there exists $s \in[0, L !)$ such that the sum $d+s$ is relatively prime to each of the numbers $1,2, \ldots, L$.

At stage $N$ : Let $\delta_{N}$ be a positive number sufficiently small that

$$
\delta_{N}<\left(\frac{1}{h_{N}}\right)^{3} \text { and }\left(\delta_{N+1}+\delta_{N+2}+\cdots\right)<\delta_{N}
$$

where this latter condition may be obtained inductively by, for example, insuring that each $\delta_{n}$ is less than $\frac{1}{2} \delta_{n-1}$. Pick the $b$ of figure 1.1 sufficiently large that

$$
\frac{\mu\left(B_{N} \Delta T^{h_{1}}\left(B_{N}\right)\right)}{\mu\left(B_{N}\right)}<\delta_{N} / 2 N h_{N} .
$$

Finally, set $f_{n} \triangleq\left[N \cdot\left(h_{1}+\cdots+h_{N}\right)\right]$ ! and pick an $s \in\left[0, f_{N}\right)$ such that

$$
h_{N+1} \text { is relatively prime to each of } 1,2, \ldots, N \cdot\left(h_{1}+\cdots+h_{N}\right)
$$

A certain weak limit $S_{\dot{c}}$. Pick some bound $M$ and a vector of integers

$$
\vec{c} \triangleq\left\langle c_{1}, c_{2}, \ldots\right\rangle \in \mathbb{Z}^{\mathbb{N}}
$$

such that $\left|c_{n}\right|<M$ for each $n$. Set $r_{n} \triangleq c_{n} h_{n}$. In passing, note that $\left\{r_{n}\right\}_{1}^{\infty}$ is a sequence of rigidity times for $T$ since $\left\{h_{n}\right\}_{1}^{\infty}$ is, and the $c_{n}$ are bounded. Let $l_{n}$ denote the sum $r_{1}+r_{2}+\cdots+r_{n-1}$. Define a transformation

$$
S_{\bar{c}} \stackrel{\unlhd}{=} \lim _{n \rightarrow \infty} T^{\prime n} .
$$

Does this limit exist? Fix some $N>M$. Pick an $n \geq N$ and let $B$ denote the set $B_{n}$. Assuming, for notational convenience, that $c_{n}$ is positive

$$
\mu\left(B \Delta T^{r_{n}} B\right) \leq \sum_{j=1}^{c_{n}} \mu\left(T^{(j-1) h_{n} B} \Delta T^{j h_{n}} B\right)<c_{n} \cdot \mu\left(B \Delta T^{h_{n} B}\right) .
$$

This latter term, by (1.4), is less than $M \cdot \mu(B) \delta_{n} / 2 n$ and hence

$$
\mu\left(B \Delta T^{r} B\right)<\frac{1}{2} \delta_{n} \mu(B)
$$

where, recall, $B$ means $B_{n}$. Hence this inequality holds for $B$ representing any translate $T^{i} B_{n}$ and thus for $B$ any disjoint union of levels of the $n$-stack. But $B_{N}$ is such a union. The above thus holds when $B \triangleq T^{\prime \prime}\left(B_{N}\right)$, which then yields

$$
\mu\left(T^{l_{n}}\left(B_{N}\right) \Delta T^{l_{n+1}}\left(B_{N}\right)\right)<\frac{1}{2} \delta_{n} \mu\left(B_{N}\right) .
$$

Finally, we sum over $n$ and apply (1.3)

$$
\sum_{n=N}^{\infty} \mu\left(T^{l_{n}} B_{N} \Delta T^{l_{n+1}} B_{N}\right)<\frac{1}{2}\left[\delta_{N}+\sum_{n=N+1}^{\infty} \delta_{n}\right] \mu\left(B_{N}\right)<\delta_{N} \mu\left(B_{N}\right)
$$

Since this sum is finite, $\left\{T^{l_{n}} B\right\}_{n=1}^{\infty}$ is a convergent sequence of sets, when $B=B_{N}$. Hence this sequence is convergent when $B$ is a union of levels in the $N$-stack. But this holds for every $N$ greater than $M$ and since such unions generate the sigmaalgebra we get the desired conclusion that $\lim _{n \rightarrow \infty} T^{l^{\prime}}$ exists. Hence we can restate 
(1.5) as

$$
\mu\left(T^{l_{N}}\left(B_{N}\right) \Delta S_{\vec{c}}\left(B_{N}\right)\right)<\delta_{N} \mu\left(B_{N}\right) .
$$

$S_{\vec{c}}$ is rank-1. If $l_{n}=0$ for all large $n$ then $S_{\vec{c}}$ is the identity transformation. We assume henceforth that $l_{n} \neq 0$ infinitely often. So, without loss of generality (if necessary, by replacing $\vec{c}$ by $-\vec{c}$ ) we may assume $l_{n}>0$ for infinitely many $n$.

Denote $S_{\vec{c}}$ by $S$. We invoke the ' $\varepsilon$-refining' definition to show $S$ rank-1: Given any partition $Q$, any $\varepsilon>0$, there exists a Rohlin stack for $S$ such that $Q$ is $\varepsilon$-refined by the partition whose atoms are the column levels, and the complement, of this Rohlin stack. Since $T$ itself is rank-1, it suffices to exhibit the following, for each positive $\varepsilon$.

For arbitrarily large $N$ there exists a set $A^{\prime} \subset B_{N}$ and a permutation $\pi:\left[0, h_{N}\right) \rightarrow\left[0, h_{N}\right)$ such that

(i) $\mu\left(B_{N} \sim A^{\prime}\right)<\mu\left(B_{N}\right) \cdot \varepsilon$.

(ii) For each $i \in\left[0, h_{N}\right): S^{i}\left(A^{\prime}\right) \subset T^{\pi(i)}\left(B_{N}\right)$.

For then, the sets $A^{\prime}, S\left(A^{\prime}\right), \ldots, S^{h^{-1}}\left(A^{\prime}\right)$ form a Rohlin stack for $S$ which is $\varepsilon$-close to the $N$-stack of $T$.

We show that $S$ satisfies (1.6) in two steps. First, we pick a large $N$ and show $T^{l_{N}}$ rank-1; this gives a permutation $\pi$ and a stack for $T^{l_{N}}$. Then since $S$ is close to $T^{l_{*}}$, we can shave this stack a little to obtain a stack for $S$.

Fix some large $N, N>M$, such that $l_{N}>0$. Let $l$ denote $l_{N}$.

Obtaining the permutation. Suppress the subscript and write $h_{N}, B_{N}$, and $\delta_{N}$ as $h$, $B$, and $\delta$. Since $N-1 \geq M$ we have that $l \leq(N-1)\left(h_{1}+\cdots+h_{N-1}\right)$ and so $\left(1.2^{\prime}\right)$ yields that $h$ is relatively prime to $l$ and that $h>l$. Now from $(1.4), \mu\left(B \sim T^{-h} B\right)<$ $\mu(B) \delta / h$. Thus

$$
\mu(B \sim A)<\mu(B) \cdot(l-1) \delta / h<\mu(B) \cdot \delta
$$

where $A \triangleq \bigcap_{k=0}^{l-1}\left[T^{-h}\right]^{k}(B)$. The sets $A, T^{h}(A), \ldots, T^{(l-1 / h}(A)$ are all contained in $B$. So for any $i \in[0, l \cdot h), T^{i}(A)$ is contained in $T^{j}(B)$ where $j \triangleq(i \bmod h)$. Hence for each $i \in[0, h)$

$$
\left[T^{l}\right]^{i}(A) \subset T^{\pi(i)}(B)
$$

where $\pi(i) \triangleq(i l \bmod h)$. Moreover, since $l$ is relatively prime to $h, \pi(\cdot)$ is a permutation of $[0, h)$ as desired.

N.B. Viewing the number $l$ as fixed, the above paragraph holds for any large $N$ and so we have incidentally shown $T^{\prime}$ to be rank-1: Condition (1.6) is fulfilled with the roles of $S, A^{\prime}$, and $\varepsilon$ played by $T^{l}, A$, and $\delta$.

Shaving the stack. Since $T^{l}$ is close to $S$ in the weak topology, the set $A$ is almost the base of a Rohlin stack for $S$. Recall from $\left(1.5^{\prime}\right)$ that $\mu\left(S(B) \Delta T^{\prime}(B)\right)<\delta \mu(B)$. Since $S$ commutes with $T^{\prime}$, an induction argument yields

$$
\begin{aligned}
\mu\left(S^{i} B \Delta\left[T^{l}\right]^{i} B\right) & \leq \mu\left(S S^{i-1} B \Delta S\left[T^{l}\right]^{i-1} B\right)+\mu\left(S\left[T^{l}\right]^{i-1} B \Delta T^{l}\left[T^{l}\right]^{i-1} B\right) \\
& =\mu\left(S^{i-1} B \Delta\left[T^{i}\right]^{i-1} B\right)+\mu\left(S(B) \Delta T^{l}(B)\right) \\
& \leq(i-1) \cdot \delta \mu(B)+\delta \mu(B) \leq i \cdot \delta \mu(B) .
\end{aligned}
$$


Now set

$$
A^{\prime} \stackrel{\bigcap^{h-1}}{=} S_{i=0}^{-i}\left(\left[T^{i}\right]^{i} A\right) .
$$

Then, for $i \in[0, h): S^{i}\left(A^{\prime}\right) \subset\left[T^{l}\right]^{i} A \subset T^{\pi(i)} B$. Also, from the inequality above and (1.7)

$$
\begin{aligned}
\mu\left(B \sim S^{-i}\left[T^{l}\right]^{i} A\right) & =\mu\left(S^{i} B \sim\left[T^{l}\right]^{i} A\right) \\
& \leq \mu\left(S^{i} B \sim\left[T^{l}\right]^{i} B\right)+\mu(B \sim A) \\
& \leq \mu(B)[i \delta+\delta]<\mu(B) / h^{2}
\end{aligned}
$$

where this last inequality follows, since $i<h$, by recalling that $\delta<1 / h^{3}$. Thus

$$
\begin{aligned}
\mu\left(B_{N} \sim A^{\prime}\right) & \leq \sum_{i=0}^{h-1} \mu\left(B \sim S^{-i}\left[T^{l}\right]^{i} A\right) \\
& \leq h \cdot \mu(B) / h^{2}=\mu\left(B_{N}\right) \cdot\left(1 / h_{N}\right)
\end{aligned}
$$

where we have rematerialized the $N$ in the first and last expressions. Since $N$ could have been chosen sufficiently large to insure that $\left(1 / h_{N}\right)$ is smaller than a given $\varepsilon$, criterion (1.6) assures $S$ rank-1.

Defining the group $G$. Let $\vec{G} \subset \mathbb{Z}^{\mathbb{N}}$ be the set of all bounded vectors; $\vec{c}=\left\langle c_{1}, c_{2}, \ldots\right\rangle$ is in $\vec{G}$ if there is some $M$ such that $\left|c_{n}\right|<M$ for all $n . \vec{G}$ is a group under addition of vectors and so

$$
G \triangleq\left\{S_{\vec{c}}: \vec{c} \in \vec{G}\right\}
$$

is a subgroup of $C(T)$. For any $\vec{c}$ such that the corresponding sum sequence $\left\{l_{n}\right\}$ is not 'eventually zero', we showed $S_{\vec{c}}$ to be rank-1. Conversely, if the $\left\{l_{n}\right\}$ are 'eventually zero' then $S_{\vec{c}}$ is the identity. Thus each $S \in G \sim\{\mathrm{Id}\}$ is rank-1.

$G$ is the image of $\vec{G}$ under the group homomorphism $\vec{c} \mapsto S_{\vec{c}}$. Its kernel is the set of $\vec{c}$ such that $\left\{l_{n}\right\}$ is eventually zero; this forces $\left\{c_{n}\right\}$ to be eventually zero. Hence the kernel of the homomorphism is countable. Since $\vec{G}$ is uncountable, $G$ is forced to be uncountable as well, completing the proof.

\section{Uncountable weak essential commutant}

For a rank-1 transformation $T,|\mathrm{WEC}(T)|=1$ where, recall, the weak essential commutant is $C(T)$ modulo the closure of the powers of $T$. When $T$ is mixing one can show, $[4]$, that

$$
|\mathrm{WEC}(T)| \leq \mathrm{rk}(T) \text {. }
$$

In this section we show that with the hypothesis of mixing relaxed to weak mixing, the inequality may fail by producing a rank- $2 T$ with uncountable weak essential commutant.

Definition 2.1. Given a transformation $B: X \rightarrow X$ and a compact group $G$ equipped with Haar measure, recall that a group extension $T$ is a transformation on $X \times G$ (endowed with product measure) determined by a choice of cocycle, a measurable map $c: X \rightarrow G$. Writing $c(x)$ as $c_{x}$, the action of $T$ on $x, h \in X \times G$ is

$$
x, h \stackrel{T}{\mapsto} B(x), h c_{x}
$$

where juxtaposition $h c_{x}$ indicates multiplication in $G$. 
There is a canonical copy, $\hat{G}$, of $G$ in $C(T)$ defined by

$$
x, h \stackrel{\hat{g}}{\mapsto} x, g h
$$

for each $g \in G$. Associativity of the group multiplication implies $\hat{g}$ commutes with $T$. Note that $\hat{g} \hat{h}=\widehat{g h}$.

Recall that the center of a group $G$ is the subgroup of those elements $g \in G$ which commute with every element.

LEMMA 2.2. If $T$ is a group extension of $B$ by $G$ then

$$
\mid \text { WEC }(T)|\geq| G / \text { Center }[G] \mid \text {. }
$$

Proof. There are three canonical group homomorphisms $\alpha, \beta$, and $\gamma$ :

$$
\begin{aligned}
C(T) / \mathrm{WCl}(T) \stackrel{\alpha}{\rightarrow} C(T) / \text { Center }[C(T)] & \hat{\uparrow} \\
& \hat{G} /(\hat{G} \cap \operatorname{Center}[C(T)]) \stackrel{\gamma}{\rightarrow} \hat{G} / \operatorname{Center}[\hat{G}] .
\end{aligned}
$$

Since $\alpha$ and $\gamma$ are surjective, and $\beta$ is injective, the size of the quotient groups decrease from upper left to lower right.

In light of the lemma, it suffices to build a rank-2 $T$ as a group extension by an uncountable group $G$ with trivial center. It will be convenient to produce this $G$ as a projective limit of finite groups.

Definition. The standard dihedral group $D_{m}$ is the group of symmetries of an $m$-sided regular polygon. Evidently it is generated by a rotation $r$ and a flip $f$ such that

$$
r^{m}=1 \quad f^{2}=1 \quad r f=f r^{-1}
$$

(where 1 denotes the identity element) and is the free group on $\{r, f\}$ with these relations.

For an arbitrary abelian group $A$ (written additively with 0 as the identity) we define a group dihedral of $A$, written $\mathscr{D}(A)$, as a semidirect product

$$
\mathscr{D}(A) \triangleq A \times\{-1,1\}
$$

with the following multiplication. For $a, b \in A$ and $i, j \in\{-1,1\}$

$$
\langle a, i\rangle\langle b, j\rangle \triangleq\langle a+i b, i j\rangle \text {. }
$$

The element $\langle 0,-1\rangle$ filips you between the two copies of $A$ in $\mathscr{D}(A)$. Note that $D_{m}$ is $\mathscr{D}\left(\mathbb{Z}_{M}\right)$.

Elements in the center of $\mathscr{D}(A)$ come from (non-trivial) elements of order 2, that is, elements $a \in A \sim\{0\}$ with $a+a=0$. One verifies

$$
\begin{aligned}
\mathscr{D}(A) \text { abelian } & \Leftrightarrow \text { Every element of } A \text { is of order } 2 . \\
\text { Center }[\mathscr{D}(A)] \text { trivial } & \Leftrightarrow \text { No element of } A \text { is of order } 2 .
\end{aligned}
$$

The construction. It will be convenient to initially do the counterexample with a finite group for $G$. Fix an $M \in \mathbb{N}$. Agree to write the elements 0 and 1 of $\mathbb{Z}_{M}$ as $\overrightarrow{0}$ and $\overrightarrow{1}$. Define two elements $r$ and $f$ in $D_{M}=\mathscr{D}\left(\mathbb{Z}_{M}\right)$

$$
r \stackrel{\unlhd}{\triangleq}\langle\overrightarrow{1}, 1\rangle \quad f \triangleq\langle\overrightarrow{0},-1\rangle,
$$


$\mathbb{Z}_{M}$ is a subgroup of $D_{M}$ with two cosets. Set $G \unlhd D_{M}$. Given a $g \in G$, the map $g \mapsto g f$ flips you from one coset to the other. The action $g \mapsto g r$, applied repeatedly, cycles you through all the elements of whichever coset you are in.

The base transformation $B$. Define a rigid rank-1 $B: X \rightarrow X$ as follows, where $b_{n} \rightarrow \infty$.

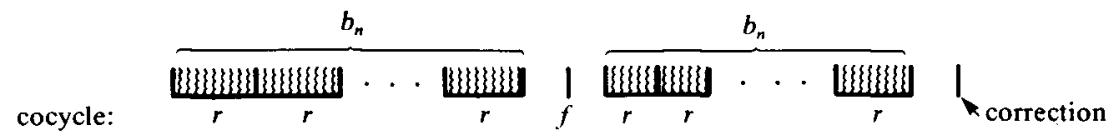

FIGURE 2.4. The $(n+1)$-block consists of $b_{n}$ many $n$-blocks, then a 'flip' spacer, followed by $b_{n}$ additional $n$-blocks and finally a 'correction' spacer.

On $X$, inductively define a partition $P$ with countably many letters. At stage $n+1$, assign two never-before-used letters to the two spacers in the above figure. This $P$ evidently generates under the action of $B$.

With $h_{n}$ denoting the height of the $n$-stack let $W_{i}^{n}$, for $i \in\left[0, h_{n}\right)$, denote the $i$-th level of the stack. Since each $W_{i}^{n}$ is a subset of a letter of $P$, we view it also as labelled with this letter. The $n$-block is thus the word $W_{0}^{n} W_{1}^{n} \cdots W_{h_{n}-1}^{n}$.

We define a cocycle $c: X \rightarrow G$ which is constant on each level of the $n$-stack. Consequently one may write $c\left(W_{i}^{n}\right)$ to indicate the value of the cocycle on (any point $x$ of) $W_{i}^{n}$. We want that the cocycle's product along the $n$-block is the rotation $r$. That is,

$$
c\left(W_{0}^{n}\right) \cdot c\left(W_{1}^{n}\right) \cdot \ldots \cdot c\left(W_{h_{n}-1}^{n}\right)=r
$$

(where here, as we will occasionally do in the future, we have used a dot $\cdot$ to emphasize multiplication in the group). Supposing that (2.5) holds, define the cocycle on the two spacers of the $(n+1)$-block by

$$
c \text { ('flip spacer') } \triangleq f
$$

and then let $c$ ('correction spacer') be the element of the group so that $(2.5)$ holds for the $(n+1)$-block.

Lastly, our transformation $T: X \times G \rightarrow X \times G$ is to be the skew product mapping

$$
x, g \mapsto B(x), g c_{x}
$$

where $c_{x}$ denotes $c(x)$.

The rank of $T$ is less than or equal to 2 . By abuse of notation, use the symbol $D_{M}$ to denote the partition of $G$ into its $2 M$ elements. Then $P \times D_{M}$ is a generating partition for $T$.

Fix $n$ and suppress it from the notation e.g., write $W_{i}^{n}$ as $W_{i}$. Suppose $x$ is in the base of the $n$-stack, $x \in W_{0}$. For a $g \in G$, the $T, P \times D_{M}$-h-name of the point $x, g$ is the sequence of letter-pairs

$$
\left(W_{0}, g_{0}\right)\left(W_{1}, g_{1}\right) \cdots\left(W_{h-1}, g_{h-1}\right)
$$

where $g_{0} \triangleq g$ and $g_{i+1} \triangleq g_{i} \cdot c\left(W_{i}\right)$. Since $r$ is the product of the cocycle along the $n$-block, $g_{h}=g \cdot r$.

Denote the $P \times D_{M}$-word (2.6) by [ $g$ ]. We exhibit two $P \times D_{M}$-words which cover most of any particular $T$-name. Most of a $T$-name is covered by $(n+1)$-blocks. Let 
$x, g$ be a point along this orbit with $x$ in the base of the $(n+1)$-stack. As $x$ traverses the first $b\left(=b_{n}\right)$ blocks of figure 2.4 the $T, P \times D_{M}$-name of $x, g$ is

$$
[g][g r]\left[g r^{2}\right] \cdots\left[g r^{b-1}\right] .
$$

We may have chosen $n$ sufficiently large that $b_{n} \gg M$. Recall that $D_{M}$ is a union of two cosets, $\mathbb{Z}_{M}$ and $f \cdot \mathbb{Z}_{M}$. If $g$ is an element of the latter then there is an $i<M$ such that $g r^{i}=f$. Thus discarding the first $i$ terms of (2.7) we see that the rest of it (except for the last at-most- $M$ terms) is covered by contiguous copies of the 'rotation-word'

$$
R(f) \triangleq[f][f r]\left[f r^{2}\right] \cdots\left[f r^{M-1}\right] .
$$

Conversely, if $g$ is an element of the other coset, then contiguous copies of the rotation-work $R\left(1_{G}\right)$ cover most of $(2.7)$, where $1_{G}$ denotes the identity element of $G$. Since these two rotation-words cover most of the $(n+1)$-block, $\operatorname{rk}(T) \leq 2$.

Note in passing that if $M>2$ then $D_{M}$ is not abelian and, since $C(T)$ non-abelian prohibits $T$ rank-1, the rank of $T$ is exactly 2 . Moreover, if $M$ is odd, forcing the center of $D_{M}$ to be trivial, then

$$
|\mathrm{WEC}(T)| \geq\left|D_{M}\right|=2 M
$$

by the preceding lemma.

$T$ can be made weak mixing. Due to the flip spacer of the $(n+1)$-block, about half of the $(n+1)$-block is covered by one rotation-word and half by the other. Since a fixed percentage (independent of $n$ ) of the $n$-block is covered by each of the two rotation-words at stage $n, T$ is ergodic.

To obtain weak mixing we kill off any non-one eigenvalue by means of the standard argument for Chacón's transformation. Modify the block structure of $T$ so that in figure 2.4 each of the two groups of $b_{n} n$-blocks is replaced by the pattern below, with $b_{n}^{\prime} \rightarrow \infty$. This affects none of the properties of $T$ so far established.

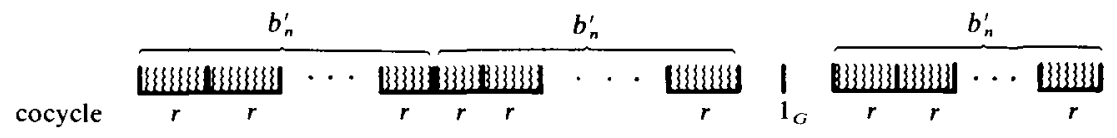

FigURE 2.8. The replacement pattern: A 'clump' of $b_{n}^{\prime}$ many $n$-blocks, followed by another clump, then a 'weak mixing' spacer, and finishing with another clump.

Suppose the $T$-name of a point enters the leftmost clump of the figure on element, say, $g \in G$. If the orbit must then enter each of the remaining two clumps also on the element $g$, then any non-one eigenvalue is prohibited and weak mixing is established. But this orbit property is easy to obtain by arranging that, for large $n$, $b_{n}^{\prime}$ is a multiple of $M$.

Making the group $G$ uncountable. Getting specific, set $b_{n}^{\prime} \triangleq n$ ! for the base transformation $B$. Our group extension of $B$ by $D_{M}$ we henceforth denote by $T_{M}$. All the $\left\{T_{m}\right\}_{m=1}^{\infty}$ have $B$ as a common factor and so we consider making a group extension $T$ as a projective limit along some of the $\left\{T_{m}\right\}$. 
There is a canonical group homomorphism from $\mathbb{Z}_{2 m}$ onto $\mathbb{Z}_{m}$ and consequently from $D_{2 m}$ to $D_{m}$. Let $G$ be the projective limit

$$
G \stackrel{\unlhd}{=}\left(\cdots \rightarrow D_{8} \rightarrow D_{4} \rightarrow D_{2} \rightarrow D_{1}\right) .
$$

Alternatively, $G$ can be described as $\mathscr{D}(A)$ where $A$ is the familiar dyadic adding machine group

$$
A \unlhd\left(\cdots \rightarrow \mathbb{Z}_{8} \rightarrow \mathbb{Z}_{4} \rightarrow \mathbb{Z}_{2} \rightarrow \mathbb{Z}_{1}\right)
$$

This $A$ can be written as $\{0,1\}^{\mathbb{N}}$, the set of binary vectors $\vec{a}=\left(\cdots a_{2} a_{1} a_{0}\right)$ with $a_{n} \in\{0,1\}$, endowed with 'addition with carry': If $\vec{c}=\vec{a}+\vec{b}$ then $c_{n}$ is the sum $a_{n} \oplus b_{n} \oplus$ carry $(n)$ in $\mathbb{Z}_{2}$, where carry $(n)$ denotes the binary carry from the $(n-1)$ place. Let $\overrightarrow{0} \unlhd(\cdots 00)$ denote the identity element. The subgroup generated by the element $\overrightarrow{\mathbf{l}} \triangleq(\cdots 001)$ is a dense copy of $\mathbb{Z}$ in $A$. Equipping $A$ with Haar measure, the group rotation

$$
\vec{a} \mapsto \vec{a}+\overrightarrow{1}
$$

is the familiar adding machine transformation, which is rank-1.

Exactly as before, define $T$ as the group extension of $B$ by $G$ determined by the cocycle of figures 2.4 and 2.8 where $r, f \in G$ are

$$
r \triangleq\langle\overrightarrow{1}, 1\rangle \quad f \triangleq\langle\overrightarrow{0},-1\rangle
$$

as before. Choose some positive integer $k$ and set $m \unlhd 2^{k}$. By abuse of notation we interpret the symbol $D_{m}$ as a partition of $G$ into $2 m$ many atoms; $\langle\vec{a}, j\rangle$ and $\left\langle\vec{b}, j^{\prime}\right\rangle$ are in the same atom if $j=j^{\prime}$ and $a_{i}=b_{i}$ for $0 \leq i<k$. This partition, being the cosets of the kernel of the group homomorphism $G \rightarrow D_{m}$, is invariant under group rotation. Thus, letting $\mathscr{B}$ denote the sigma-algebra of the base transformation $B$

$$
\mathscr{F}_{k} \leqq \mathscr{B} \times D_{m} \quad\left(\text { where } m=2^{k}\right)
$$

is a factor algebra of $T$. The factor transformation $T \mid \mathscr{F}_{k}$ is evidently $T_{m}$. Also,

$$
\mathscr{F}_{1} \subset \mathscr{F}_{2} \subset \mathscr{F}_{3} \subset \cdots \text { and } \bigvee_{k=1}^{\infty} \mathscr{F}_{k}=\text { The whole sigma-algebra. }
$$

$T$ is rank-2 and weak mixing. That $\mathrm{rk}(T) \leq 2$ follows on general principles from the fact that each $T_{m}$ has its rank so bounded. For any transformation $T$ with factor algebras as in (2.9)

$$
\operatorname{rk}(T)=\sup _{k} \operatorname{rk}\left(\left.T\right|_{\mathscr{F}_{k}}\right) \text {. }
$$

This follows from the ' $\varepsilon$-refining' characterization of rank given in $\S 0$ and is proved in $[4 ; \S 1]$. Sketching the proof, it suffices to show that $r \leq \operatorname{rk}(T)$ where $r$ is the value of the above supremum. Given $\varepsilon$ and a (finite) partition $Q$, choose a large $k$ and a partition $Q^{\prime} \subset \mathscr{F}_{k}$ such that $\left|Q-Q^{\prime}\right|<\varepsilon$ in the symmetric difference metric. Since rk $\left(T \mid \mathscr{F}_{k}\right) \leq r$, this $Q^{\prime}$ can be $\varepsilon$-caught by the column levels of some $r$ disjoint stacks in $\mathscr{F}_{k}$; hence $Q$ is $2 \varepsilon$-caught by them. Thus rk $(T) \leq r$.

The adding machine group, $A$, has no elements of finite order and hence $G=\mathscr{D}(A)$ has trivial center. Thus $T$ is not rank-1 since $C(T)$, as it contains $G$, is non-abelian; so $\mathrm{rk}(T)=2$. By lemma $2.2,|\operatorname{WEC}(T)| \geq|G|$ and so WEC $(T)$ is uncountable.

Because each $T_{m}$ is weak mixing, we may conclude the same for $T$ on general principles: 
LEMMA 2.10. If $\overline{\mathscr{F}_{1}} \subset \mathscr{F}_{2} \subset \cdots$ is a tower of factor algebras whose join generates for a transformation $T$, then if each $\left.T\right|_{\mathscr{F}_{k}}$ is weak mixing, $T$ is weak mixing.

Proof. Suppose $T f=\lambda \cdot f$ where $f$ is an eigenfunction with eigenvalue $\lambda$. Let $T_{k}$ denote the factor transformation $\left.T\right|_{\mathcal{F}_{k}}$ and let $f_{k}$ be the conditional expectation $\mathscr{E}\left(f \mid \mathscr{F}_{k}\right)$. Then

$$
T_{k} f_{k}=\mathscr{E}\left(T f \mid \mathscr{F}_{k}\right)=\mathscr{E}\left(\lambda f \mid \mathscr{F}_{k}\right)=\lambda \cdot f_{k} .
$$

So $f_{k}$ is an eigenfunction for $T_{k}$ and thus is constant. By the Martingale convergence theorem, $f_{k} \rightarrow f$ a.e. and so $f$ is constant. This completes the lemma and consequently the counterexample of this section.

Remark. The example could have been made using $G$ of the form $\mathscr{D}(A)$ for $A$ the circle group or, indeed, any compact abelian group possessing a group rotation which is rank-1. One can show directly that $\mathrm{rk}(T) \leq 2$ without resorting to a tower of factor algebras. We chose to use a tower of factors so as to facilitate showing $T$ weak mixing.

Question. When $T$ is of finite rank, WEC ( $T$ ) need not be a finite group. Can it be non-compact? What conditions on $T$ imply that $\mathrm{WCl}(T)=$ Center $[C(T)]$ or that $C(T) /$ Center $[C(T)]$ is compact?

\section{A non-monotone rank function and an invariant}

Using a generalization of group extension this section produces an example in the class 'weak mixing rank-1' of a transformation $S$ where the rank function

$$
l \mapsto \operatorname{rk}\left(S^{l}\right)
$$

is oscillatory; this partially answers a question raised in [3]. The construction suggests an invariant $\mathscr{L}(\cdot)$ which associates to each rigid rank-1, a semigroup in $\mathbb{N}$. As a byproduct, certain prescribed roots can be prohibited. The technique appears to have a novel feature: $l$ th roots are killed off by proving a property of the $l$ th power of the transformation, namely, that $S^{l}$ has non-abelian commutant.

Definition. Given a transformation $B: X \rightarrow X$ and a compact group $G$ endowed with Haar measure, an automorphism extension $S: X \times G \rightarrow X \times G$ is determined by choosing a measurable group automorphism $\alpha \in$ Aut $(G)$ and map $e: X \rightarrow G$. $S$ acts via

$$
x, h \stackrel{s}{\mapsto} B(x), \alpha(h) \cdot e_{x}
$$

where we have written $e_{x}$ for $e(x)$. The uniqueness of the invariant probability measure on $G$ forces it to be $\alpha$-invariant; product measure is consequently preserved by $S$. Iterating the transformation yields

$$
x, h \stackrel{S^{n}}{\mapsto} B^{n}(x), \alpha^{n}(h) \cdot c_{x}
$$

where

$$
c_{x} \stackrel{\unlhd}{=} \alpha^{n-1}\left(e_{x}\right) \cdot \alpha^{n-2}\left(e_{B x}\right) \cdot \ldots \cdot \alpha\left(e_{B^{n-2} x}\right) \cdot e_{B^{n-1} x} .
$$

Unlike the case of a group extension, an automorphism extension does not necessarily have a copy of $G$ in the commutant and indeed $C(S)$ can be trivial. However if $G$ is finite then there is a positive integer $A$ (the smallest such is called 
the order of $\alpha$ ) such that $\alpha^{A}$ is the identity automorphism. Setting $n \unlhd A$ in (3.2) shows that $S^{A}$ is a group extension of $B^{A}$ by $G$ via the cocycle (3.2'). Thus $\hat{G}$ is contained in $C\left(S^{A}\right)$ where, recalling (2.1), for $g \in G$ the 'multiplication on the left' transformation $\hat{g}$ sends $x, h$ to $x, g h$. More generally, for an integer $n$ :

$$
\hat{g} \in C\left(S^{n}\right) \Leftrightarrow g \text { is a fixed point of } \alpha^{n} .
$$

Here is the game plan: Build an automorphism extension $S$ by $\mathbb{Z}_{M}$ such that $S^{\text {odd }}$ can be shown to be rank-1, directly, by exhibiting a word which covers most of a name. Then show that $\mathrm{rk}\left(S^{\text {even }}\right) \geq 2$, indirectly, by showing $C\left(S^{\text {even }}\right)$ non-abelian. Non-abelianness will follow by the existence of a $g \in G$ which is a fixed point of $\alpha^{\text {even }}$ but not of $\alpha$.

Actually we do the construction slightly more generally. The above overview describes the result of the following construction when $M=4, A=2$, and $\alpha$ is the automorphism of $\mathbb{Z}_{4}$ which sends 1 to -1 .

Let $n \perp m$ mean that $n$ and $m$ are relatively prime. $\mathbb{Z}_{n}$ or $\mathbb{Z}(n)$ will denote the cyclic group $\mathbb{Z} / n \mathbb{Z}$.

Construction. Choose an $M$ and let our group be $G \unlhd \mathbb{Z}_{M}$. Let $r \in G$ be a synonym for $1 \in \mathbb{Z}_{M}$; we use this alternate name because we wish to write $G$ multiplicatively, with $1_{\mathrm{G}}$ denoting the identity element. Pick a non-identity $\alpha \in$ Aut $(G)$ and let $A$ denote its order. Cut and stack a transformation $B: X \rightarrow X$ inductively according to the following figure.

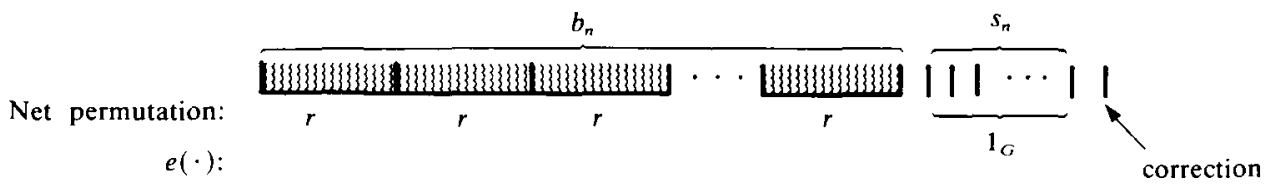

FIGURE 3.3. The $n$-block of $B: b_{n}(n-1)$-blocks followed by $s_{n}+1$ spacers. We require $b_{n} \rightarrow \infty$.

Let $S$ be our automorphism extension (3.1). The map $e(\cdot)$ is to be constant on the column levels of the $n$-stack; it is the identity element on all spacers but the last.

For $S$ to be defined on a probability space, the summability condition

$$
\sum_{n=1}^{\infty} \frac{s_{n}+1}{h_{n}}<\infty
$$

must be satisfied. It turns out that (at stage $n$, with $h_{n-1}$ known) the conditions to follow ask that $h_{n}$ be some value, modulo another. Hence we have an infinite list from which to choose $h_{n}$. Choosing one sufficiently large that $h_{n-1} / h_{n}<2^{-n}$, we then choose $b_{n}$ and $s_{n}, s_{n}<h_{n-1}$, so that $b_{n} h_{n-1}+s_{n}+1$ equals $h_{n}$. The sum in (3.4) is now less than 1 .

Making $S$ rank-1. We traverse a long string of contiguous $n$-blocks. Suppose we knew that, if we enter the first $n$-block on $g$, then we enter the second on $g r$, the third on $g r^{2}$, and so on. Since $r^{M}=1_{G}$, we can emulate $\S 2$ and make an $M h_{n}$-word, a 'rotation-word', which covers most of the $(n+1)$-block; this insures $S$ rank-1. 
So we want that the net effect of traversing an $n$-block (the net permutation on $G$ ) is multiplication by $r$. For this, $\alpha^{h_{n}}$ must, in light of (3.2), be the identity automorphism. This requires

$$
h_{n} \equiv 0 \quad(\bmod A) .
$$

Conversely, given this we can define $e$ ('correction') in (3.3) so that the net permutation along the $n$-block is indeed 'multiplication by $r$ '. Let $h_{n}^{\prime} \triangleq M h_{n}$ denote the length of the consequent rotation-word which covers, by contiguous copies, most of the $(n+1)$-block.

Obtaining rank-1 for certain powers. For which $l$ can $S^{l}$ be rank-1? In order to imitate the argument of $\S 1$ showing powers to be rank-1, we need that $l \perp h_{n}^{\prime}$ for large $n$; conversely, if this condition holds then, since $b_{n} \rightarrow \infty, S^{l}$ is forced to be rank-1.

The only way an $l$ could be relatively prime to an $h_{n}^{\prime}$, in view of $(3.5)$, is if $l \perp A \cdot M$; call $l$ good if this holds.

We need to provide an infinite list from which to choose $h_{n}$. Evidently any

$$
h_{n} \in\left\{A^{k}\right\}_{k=1}^{\infty}
$$

works, satisfying (3.5) and making $h_{n}^{\prime}$ relatively prime to exponents which are good. Thus any $S^{\text {good }}$ is rank-1.

Incorporating weak mixing into $S$. Alter $B$ by replacing the $b_{n} n$-blocks of figure 3.3 by the pattern of figure 2.8: two clumps of $b_{n}^{\prime}$ many $n$-blocks, then a spacer (on which $e(\cdot)$ is $1_{G}$ ) followed by a third clump. Since $r^{M}=1_{G}$, the Chacón argument goes through if $b_{n}^{\prime}$ can be chosen a multiple of $M$. This requires only a rewording of the summability paragraph below (3.4).

With $h_{n-1}$ known, pick an $h_{n}$ in the list such that $4 M h_{n-1} / h_{n}<2^{-n}$. The block structure described above wants $h_{n}$ to be of the form

$$
2 b_{n}^{\prime} h_{n-1}+1+b_{n}^{\prime} h_{n-1}+s_{n}+1
$$

for some choice of $b_{n}^{\prime}$ and $s_{n}$. Writing $b_{n}^{\prime}$ as $M \cdot b_{n}^{\prime \prime}$, a multiple of $M$, the expression equals

$$
\left(3 M h_{n-1}\right) b_{n}^{\prime \prime}+\left(s_{n}+2\right) .
$$

Pick natural numbers $b_{n}^{\prime \prime}$ and $s_{n}<3 M h_{n-1}$ (consequently, $s_{n}+2<4 M h_{n-1}$ ) such that (*) equals our chosen $h_{n}$. The sum

$$
\sum_{1}^{\infty} \frac{s_{n}+2}{h_{n}}<1
$$

and hence is finite, as required.

This completes the construction.

We have built a weak mixing $S$ for which $S^{l}$ is rank-1 whenever $l \perp A \cdot M$. Conversely, rk $\left(S^{l}\right) \geq 2$ whenever $C\left(S^{l}\right)$ is non-abelian. $C\left(S^{l}\right)$ contains the set

$$
\left\{\hat{g} S^{i}: \alpha^{\prime}(g)=g\right\}
$$

for $g \in G$ and $i \in \mathbb{Z}$. If $\alpha^{\prime}$ has no more fixed points than $\alpha$, then this set is abelian (since it is contained in $C(S)$ ) and tells us nothing. Contrariwise, if $g$ is fixed by 
$\alpha^{\prime}$ but not by $\alpha$, then $\hat{g}$ and $S$ are two elements of $C(S)$ which fail to commute. Thus

$$
\operatorname{rk}\left(S^{l}\right)= \begin{cases}1 & \text { if } l \perp A \cdot M \\ \geq 2 & \text { if } \exists g, \alpha^{\prime}(g)=g \neq \alpha(g) \\ \text { unknown } & \text { otherwise. }\end{cases}
$$

In particular, rk $\left(S^{l}\right) \geq 2$ when $l$ is a multiple of the order, $A$, of $\alpha$.

A judicious choice of $M$ and $\alpha$ makes the 'unknown' case, vacuous. Each automorphism $\alpha$ of a cyclic group, $\mathbb{Z}_{M}$, is determined by a choice of number in the group which is relatively prime to $M$; the action of $\alpha$ is multiplication, in the ring $\mathbb{Z}_{M}$, by this number. Suppose $M$ is of the form $p^{2}, p \in \mathbb{N}$, and let $\alpha$ be multiplication by $p+1$. The order of $\alpha$ is $p$. If we choose $p$ prime then any $l$ not relatively prime to $A M\left(=p^{3}\right)$ is in fact a multiple of $A$. Thus

Application 3.7'. For each prime p there exists a weak mixing $S$ such that

$$
\operatorname{rk}\left(S^{l}\right)= \begin{cases}1 & \text { if } l \perp p \\ \geq 2 & \text { otherwise. }\end{cases}
$$

Remark. The result is, happily, consistent with a general fact which follows from the 'name' characterization of rank:

$$
\text { rk }(T) \leq \mathrm{rk}\left(T^{l}\right) \leq l \cdot \operatorname{rk}(T), \quad l \in \mathbb{N} .
$$

The righthand inequality is useful in $\left(3.7^{\prime}\right)$ in the case $p=2$ : The rank of $S^{2 \cdot \text { odd }}$ is exactly 2 .

One is tempted to plug in other choices of $M$ and $\alpha$ into (3.7) and see what one can get for $\left\{l:\right.$ rk $\left.\left(S^{l}\right)=1\right\}$. However, the construction of $S$ puts an algebraic constraint on what one can do. This constraint is not entirely an artefact of the proof, in the following sense: The difficult part, lower bounding the rank of a power, is done by showing this power not commutant dense. There is an intrinsic algebraic constraint, part (d) of the proposition below, on the set of powers which are commutant dense.

Definition. For transformations $T$ and $S$ on the same space, let $T \rightarrow S$ or $S \leftarrow T$ indicate that $S \in \mathrm{WCl}(T)$. Viewed as a relation, $\rightarrow$ is transitive and hence $\leftrightarrow$ is an equivalence relation. On the class $C D$ of commutant dense transformations, the relations $\rightarrow, \leftrightarrow$, and 'commutes with', are evidently the same relation.

Let $\mathscr{L}(T)$ denote the subset of those $l \in \mathbb{N}$ such that $T^{\prime} \in \mathrm{CD}$.

Proposition 3.8. $T$ and $S$ denote transformations on the same space.

(a) $T \rightarrow S \Rightarrow$ [For any $\left.l: T^{l} \rightarrow S^{l}\right]$.

(b) $[S \in C D$ and $S \leftarrow T] \Rightarrow T \in C D$.

Equivalently: 'Commutant dense' is inherited under under $\leftarrow$.

(c) $\mathrm{CD}$ is closed under taking roots.

(d) $\mathscr{L}(T)$ is closed under factors and, when non-empty, is a semigroup under multiplication.

(e)-(h) deferred.

Proof (a). The group of transformations on the space form a topological group under the weak topology. 
(b) If $R \in C(T) \subset C(S)$ then $R \leftarrow S \leftarrow T$.

(c) If $T$ a root of $S$ then $T \rightarrow S$ and apply (b).

(d) If $l \in \mathscr{L}(T)$ and $m \mid l$ then, by (c), $m \in \mathscr{L}(T)$. If $l, m \in \mathscr{L}(T)$ then $T^{l} \rightarrow T$ and $T^{m} \rightarrow T$. By (a), $\left(T^{m}\right)^{l} \rightarrow T^{l} \rightarrow T$ and so $m \cdot l \in \mathscr{L}(T)$ by (b).

Let PN denote the set of prime numbers. There is a one-to-one correspondence between: semigroups $\mathscr{L}$ in $\mathbb{N}$ closed under factors - on the one hand - and subgroups $\mathbf{P}$ of $\mathrm{PN}$, on the other. The correspondence is

$$
\mathscr{L}=\{l \in \mathbb{N}: \forall p \in \mathbf{P}, l \perp p\} .
$$

For a given $\mathbf{P}$, denote the corresponding $\mathscr{L}$ by $\mathscr{L}_{\mathbf{P}}$.

In light of $(\mathrm{d})$, the following generalization of application $\left(3.7^{\prime}\right)$ suggests itself.

Non-MONOTONE THEOREM. For any $\mathbf{P} \subset \mathrm{PN}$ there exists a rigid and weak mixing transformation $S$ such that

$$
\mathscr{L}(S)=\mathscr{L}_{\mathbf{p}}=\left\{l \in \mathbb{N}: \operatorname{rk}\left(S^{l}\right)=1\right\} .
$$

Proof. $\mathbf{P}$ infinite is the interesting case; enumerate it as $\left\{p_{n}\right\}_{n=1}^{\infty}$. For $p$ denoting $p_{n}$, let $H_{n}$ be the group $\mathbb{Z}\left(p^{2}\right)$. Let $r_{n}^{\prime} \in H_{n}$ be the rotation (the element 1$)$ in $\mathbb{Z}\left(p^{2}\right)$ and let $\alpha_{n}^{\prime}$ be the automorphism 'multiplication by $p+1$ '. Let $G$ now be the direct product group with automorphism $\alpha$

$$
G \triangleq H_{1} \times H_{2} \times \cdots . \quad \alpha \triangleq \alpha_{1}^{\prime} \times \alpha_{2}^{\prime} \times \cdots .
$$

Let $r \in G$ be the element whose $n$th component is $r_{n}^{\prime}$.

It will be convenient to write $G$ as a projective limit

$$
G \stackrel{\unlhd}{=}\left(\cdots \rightarrow G_{3} \rightarrow G_{2} \rightarrow G_{1}\right)
$$

where $G_{N}$ is the direct product $H_{1} \times \cdots \times H_{N}$. Note that each $G_{N}$ is a cyclic group since the $\left\{p_{n}\right\}$ are relatively prime. Let $\alpha_{N} \in$ Aut $\left(G_{N}\right)$ denote $\alpha$ restricted to $G_{N}$. Let $\boldsymbol{A}_{N}$ denote the order, $p_{1} \cdot \ldots \cdot p_{N}$, of $\alpha_{N}$ and set $M_{N} \triangleq\left|G_{N}\right| \stackrel{\text { note }}{=}\left(\boldsymbol{A}_{N}\right)^{2}$.

We now replay Construction to build a base transformation $B: X \rightarrow X$ and map $e: X \rightarrow G$ which, using $\alpha$, yields the desired automorphism extension $S$. For any $l \notin \mathscr{L}_{\mathbf{P}}, \alpha^{l}$ has non-trivial fixed points: If $p_{n} \mid l$ then any element of $G$, non-identity in the $n$th component and identity elsewhere, is fixed by $\alpha^{l}$. Since $\alpha$ fixes nothing non-trivial, $C\left(S^{l}\right)$ is non-abelian. So it suffices to build $S$ with rk $\left(S^{l}\right)=1$ for $l \in \mathscr{L}_{\mathbf{P}}$. Say that $l$ is good if $l \in \mathscr{L}_{\mathbf{P}}$; for such, $l \perp A_{n} M_{n}$ for all $n$.

At stage $n$, with $h_{n-1}$ known and wishing to determine $h_{n}$, let the roles of $M$ and $A$ in Construction be played by $M_{n}$ and $A_{n}$. Set

$$
h_{n} \triangleq\left(A_{n}\right)^{k}
$$

with $k$ chosen sufficiently large that $4 M_{n} h_{n-1} / h_{n}<2^{-n}$ and consequently the summability condition, (3.6), holds.

As in the projective limit argument of $\S 2$, for each $n, S$ has as a factor the automorphism extension of $B$ by $G_{n}$ via the (restricted to $G_{n}$ ) maps $\alpha$ and $e$. Let $\mathscr{F}_{n}$ denote the factor algebra and $\left.S_{n} \triangleq S\right|_{\mathscr{F}_{n}}$.

Fix $N$. For any $n>N,(*)$ implies that $h_{n}^{\prime} \perp$ good and, since $A_{N} \mid A_{n}$, that $h_{n} \equiv 0$ $\left(\bmod A_{N}\right)$. Thus $\left(S_{N}\right)^{l}$ is rank-1 for good $l$. Moreover, $S_{N}$ is weak mixing since $M_{n} \mid b_{n}^{\prime}$ and $M_{N} \mid M_{n}$ evidently imply $M_{N} \mid b_{n}^{\prime}$. 
Since these properties are inherited under a generating tower of factors, $S$ is weak mixing and $\mathrm{rk}\left(S^{l}\right)=1$ for $\operatorname{good} l$.

To indicate its dependence on $\mathbf{P}$, write the $S$ of the theorem as $S_{\mathrm{p}}$. Simple inheritance properties of weak limits prohibit certain relations between the $S_{\boldsymbol{P}}$ for different $\mathbf{P}$.

Let $\mathscr{R}(T)$ denote the set of those $l \in \mathbb{N}$ for which $T$ has an $l$ th root. Recall that a power-joining, remark 0.4 , is a transformation formed by joining countably many powers of a given transformation.

Proposition 3.8 (continued)

(e) $[T \rightarrow S$ and $\mathscr{L}(S) \neq \varnothing] \quad \Rightarrow \quad \mathscr{L}(S)=\mathscr{L}(T)$.

Thus: $\mathscr{L}(\cdot)$ is inherited under $\leftrightarrow$.

(f) $[F$ a factor of $T$ and $\mathscr{L}(F) \neq \varnothing] \Rightarrow \mathscr{L}(F) \supset \mathscr{L}(T)$.

(g) $[S$ a power-joining of $T$ and $\mathscr{L}(S) \neq \varnothing] \quad \Rightarrow \quad \mathscr{L}(S) \supset \mathscr{L}(T)$.

(h) If $T \in \mathrm{CD}$ then $\mathscr{R}(T) \subset \mathscr{L}(T)$.

Proof of (e) If $\mathscr{L}(S) \neq \varnothing$ then $S \in \mathrm{CD}$ and so $T \leftarrow S$. Thus it suffices to establish $\mathscr{L}(S) \subset \mathscr{L}(T)$. Suppose $l \in \mathscr{L}(S)$. By (a), $S^{l} \leftarrow T^{\prime}$ and since 'commutant density' is inherited under $\leftarrow$, one concludes $l \in \mathscr{L}(T)$ as desired.

(f) If $l \in \mathscr{L}(T)$ then $T \leftarrow T^{l}$, so by restricting to the factor algebra, $F \leftarrow F^{l}$. If $\mathscr{L}(F) \neq \varnothing$ then $F$, consequently $F^{l}$, is in CD.

(g) Suppose $S=\left(T^{n_{1}} \times T^{n_{2}} \times \cdots: \nu\right)$ where $\nu \in$ Joi $\left(T^{n_{1}}, T^{n_{2}}, \ldots\right)$. Given that, say, 7 is in $\mathscr{L}(T)$ our goal is to show $7 \in \mathscr{L}(S)$.

Since $T^{7} \rightarrow T$ there exist exponents $\left\{m_{k}\right\}$ such that $\left[T^{7}\right]^{m_{k}} \rightarrow T$. Thus, for each $i$ : $\left[\left(T^{7}\right)^{n_{i}}\right]^{m_{k}} \rightarrow T^{n_{i}}$ as $k \rightarrow \infty$. As a consequence (stated in (0.2) in general)

$$
\left(T^{7 n_{1}} \times T^{7 n_{2}} \times \cdots: \nu\right)^{m_{k}} \rightarrow\left(T^{n_{1}} \times T^{n_{2}} \times \cdots: \nu\right) \quad \text { as } k \rightarrow \infty .
$$

In other words, $S^{7} \rightarrow S$. But $S \in$ CD since $\mathscr{L}(S) \neq \varnothing$. So by (b), $S^{7}$ is commutant dense.

(h) Suppose $S$ is an $l$ th root of $T$. Since $T \rightarrow S$ then $T^{l} \rightarrow S^{l}=T$. By (b), $l \in \mathscr{L}(T)$.

Remark 3.10. In part (f) the inclusion $\mathscr{L}(F) \supset \mathscr{L}(T)$ may be strict. One can produce a rank-1 $T$ with factors $F_{1}$ and $F_{2}$ such that $\mathscr{L}\left(F_{1}\right) \neq \mathscr{L}\left(F_{2}\right)$. For example, pick distinct primes $p_{1}$ and $p_{2}$ and let $F_{i}, i \in\{1,2\}$, denote the transformation $S$ from Application 3.7'. Set $T \triangleq F_{1} \times F_{2}$. This $T$ will be rank-1 if we mimick [3; example $v i$ ].

Let $h_{i}(n)$ denote the length of the $n$-block of $F_{i}$. By construction $h_{i}(n)$ is a power of $p_{i}$. Hence for each $n, h_{1}(n)$ and $h_{2}(n)$ are relatively prime. Let $H(n)$ denote the minimum of $h_{1}(n)$ and $h_{2}(n)$. If we simultaneously build $F_{1}$ and $F_{2}$ so that

$$
\frac{H(n+1)}{h_{1}(n) \cdot h_{2}(n)} \rightarrow \infty \quad \text { as } n \rightarrow \infty
$$

then $F_{1} \times F_{2}$ will be rank-1.

Remark 3.11. In part (g) there is equality $\mathscr{L}(S)=\mathscr{L}(T)$ in the following circumstance. Suppose

$$
S=\left(T^{n_{1}} \times T^{n_{2}} \times \cdots: \nu\right)
$$

and one of the $n_{i}\left(\right.$ or $\left.-n_{i}\right)$ is in $\mathscr{L}(T)$. Since $T^{n_{i}}$ is a factor of $S$,

$$
\mathscr{L}(T) \subset \mathscr{L}(S) \subset \mathscr{L}\left(T^{n_{i}}\right)=\mathscr{L}(T)
$$

by parts $(\mathrm{g}),(\mathrm{f})$, and (e) respectively. 
Where it is non-trivial, on the class $\mathrm{CD}$, property (e) says that $\mathscr{L}(\cdot)$ is inherited under $\rightarrow$. Thus, on the uncountable group of commuting transformations of $\S 1$, $\mathscr{L}(\cdot)$ is constant. In contrast, on the uncountable collection

$$
\left\{S_{\mathbf{P}}: \mathbf{P} \subset \mathbf{P N}\right\}
$$

of transformations (living on different spaces), $\mathscr{L}(\cdot)$ is injective. Thus, placing isomorphic copies of the $\left\{S_{\mathbf{P}}\right\}$ on a common space in any fashion, no two can commute since no two can be related by $\rightarrow$. Similarly, by (f), if $\mathbf{Q}, \mathbf{P} \subset \mathbf{P N}$ with $\mathbf{Q} \not \subset \mathbf{P}$ then $S_{\mathbf{Q}}$ cannot be a factor of $S_{\mathbf{P}}$.

Finally by $(\mathrm{h})$ together with the Non-monotone theorem,

$$
\mathscr{R}\left(S_{\mathbf{P}}\right) \subset \mathscr{L}\left(S_{\mathbf{P}}\right)=\{l \in \mathbb{N}: \forall p \in \mathbf{P}, l \perp p\} .
$$

In particular, $S_{\mathrm{PN}}$ has no roots. Part (h) may be useful for other examples: The non-existence of an $l$ th root is implied by the existence of two non-commuting elements in the commutant of the $l$ th power.

\section{§ 4. Closing remarks}

What kinds of self-joinings can a rank-1 have? The purpose of this section is simply to advertise a question raised in [3]: Is every ergodic joining a weak limit of diagonal joinings?

Definition. Recall that $T$ is rank-1 if, given any partition $Q$ and any $\varepsilon$, there exist a Rohlin stack whose column-levels and complement $\varepsilon$-refine $Q$. $T$ is said to have flat stacks if, calling the base set of the stack $B$, the 'first return to $B$ ' function is $(1-\varepsilon)$-constant: For $x \in B$, let $f(x)$ be the smallest $k>0$ such that $T^{k}(x) \in B$. The condition is that there exists such a stack with

$$
\mu\{x \in B: f(x)=h\} / \mu(B)>1-\varepsilon, \quad \text { for some } h .
$$

By altering the stack slightly one may assume this $h$ is the stack height. The class of transformations with flat stacks is closed under factors.

Definition. [Henceforth we talk only about 2-fold self-joinings of a transformation $(T: X, \mathscr{A}, \mu)$. A joining $\nu \in \mathrm{Joi}(T, T)$ is called ergodic if the transformation $(T \times T: X \times X, \mathscr{A} \times \mathscr{A}, \nu)$ is ergodic.] Any $S \in C(T)$ gives rise to a self-joining $\gamma_{s}$ called a graph joining defined on rectangles by

$$
\gamma_{S}(A \times B) \triangleq \mu\left(A \cap S^{-1} B\right) \quad A, B \in \mathscr{A}
$$

Since $\left(T \times T: X \times X, \gamma_{S}\right)$ and $(T: X, \mu)$ are isomorphic transformations, $\gamma_{S}$ is an ergodic joining when $T$ is ergodic.

A special case is a graph joining arising from a power of $T$. Agree to write the joining $\gamma_{T^{n}}(\cdot)$ as $\Delta^{n}(\cdot)$ and call it a diagonal joining.

For joinings $\left\{\nu_{n}\right\}$ and $\rho$, say that $\nu_{n} \rightarrow \rho$ if for all $A, B \in \mathscr{A}$

$$
\nu_{n}(A \times B) \rightarrow \rho(A \times B) \quad \text { as } n \rightarrow \infty .
$$

Fix some rank-1 $T$. The weak-closure theorem says that for each $S \in C(T)$ there exists $\left\{k_{n}\right\}$ such that $T^{k_{n}} \rightarrow S$. An equivalent formulation of this limit in terms of joining is that $\Delta^{k_{n}} \rightarrow \gamma_{S}$. Which suggests the question: Is it the case that

$$
\text { each ergodic } \nu \in \operatorname{Joi}(T, T) \text { is a weak limit } \Delta^{k_{n}} \rightarrow \nu
$$


for some $\left\{k_{n}\right\}$ ? For example, product measure $\mu \times \mu$ is such a weak limit when there exists a mixing sequence $\left\{k_{n}\right\}$ for $T$; this happens exactly when $T$ is weak mixing and thus, happily, when $\mu \times \mu$ is ergodic.

The collection of transformations fulfilling (4.1) is closed under factors. What does (4.1) assert for a partially mixing $T$

$$
\forall A, B: \quad \lim _{n \rightarrow \infty} \mu\left(A \cap T^{-n} B\right) \geq \alpha \cdot \mu(A) \cdot \mu(B)
$$

with parameter $\alpha \in(0,1]$ ? Clearly, product measure is absolutely continuous with respect to any non-trivial weak limit $\nu=\Delta^{k_{n}}$; so if $\nu$ is ergodic then $\nu=\mu \times \mu$. Consequently, (4.1) is equivalent to asking that $T$ have minimal self-joinings. These are not entirely uncharted waters: If $\alpha(T)>\frac{1}{2}$ then $T$ has minimal self-joinings, by [4]. Knowing only that $\alpha(T)>0$ still insures, via [3], $T$ prime and with trivial commutant.

We also have (4.1) for ergodic group rotations since, by [2], their ergodic selfjoinings are graphs.

There is another class of $T$ for which (4.1) holds, namely, when $T$ has flat stacks (we forego the proof, which is similar to $\S 3$ of [3]). This does not claim to settle (4.1) for rigid rank-1 since it would seem unlikely that all such transformations have flat stacks. However, all examples that we know of in the literature, of rigid rank-1's built by cutting and stacking, have flat stacks. In particular, (4.1) holds for the three examples cut and stacked in this paper.

The third author would like to thank the Laboratoires de Probabilités at the University of Paris-Jussieu and the University of Dijon for their hospitality while some of this work was done. The third author was partially supported by NSF grant DMS 8501519.

\section{REFERENCES}

[1] A. del Junco \& D. Rudolph. A rank one, rigid, prime, simple map. Ergod. Th. \& Dynam. Sys. 7 (1987), 229-247.

[2] A. del Junco \& D. Rudolph. On ergodic actions whose self-joinings are graphs. Ergod. Th. \& Dynam. Sys. 7 (1987), 531-557.

[3] J. L. King. The commutant is the weak closure of the powers, for rank-1 transformations. Ergod. Th. \& Dynam. Sys. 6 (1986), 363-384.

[4] J. L. King. Joining-rank and the structure of finite rank mixing transformations. Journal d'Analyse. 